\title{
TESSELLATIONS OF MODULI SPACES AND THE MOSAIC OPERAD
}

\author{
SATYAN L. DEVADOSS
}

\begin{abstract}
We construct a new (cyclic) operad of mosaics defined by polygons with marked diagonals. Its underlying (aspherical) spaces are the sets $\overline{\mathcal{M}}_{0}^{n}(\mathbb{R})$ which are naturally tiled by Stasheff associahedra. We describe them as iterated blow-ups and show that their fundamental groups form an operad with similarities to the operad of braid groups.
\end{abstract}

Acknowledgments. This paper is a version of my doctorate thesis under Jack Morava, to whom I am indebted for providing much guidance and encouragement. Thanks also go out to F. Hirzebruch, T. Januszkiewicz, and R. Scott for sharing useful insights. I am especially grateful to Jim Stasheff for bringing up questions about the blow-up arrangements and for his overall enthusiasm about this work.

\section{The Operads}

1.1. The notion of an operad was created for the study of iterated loop spaces 13. Since then, operads have been used as universal objects representing a wide range of algebraic concepts. We give a brief definition and provide classic examples to highlight the issues to be discussed.

Definition 1.1.1. An operad $\{\mathcal{O}(n) \mid n \in \mathbb{N}\}$ is a collection of objects $\mathcal{O}(n)$ in a monoidal category endowed with certain extra structures:

i) $\mathcal{O}(n)$ carries an action of the symmetric group $\mathbb{S}_{n}$.

ii) There are composition maps

$$
\mathcal{O}(n) \times \mathcal{O}\left(k_{1}\right) \times \cdots \times \mathcal{O}\left(k_{n}\right) \rightarrow \mathcal{O}\left(k_{1}+\cdots+k_{n}\right)
$$

which satisfy certain well-known axioms, $c f$. [14].

This paper will be concerned mostly with operads in the context of topological spaces, where the objects $\mathcal{O}(n)$ will be equivalence classes of geometric objects.

Example 1.1.2. These objects can be pictured as trees (Figure 17a). A tree is composed of corollas with one external edge marked as a root and the remaining external edges as leaves. Given trees $s$ and $t$, basic compositions are defined as $s \circ_{i} t$, obtained by grafting the root of $s$ to $i^{t h}$ leaf of $t$. This grafted piece of the tree is called a branch.

Example 1.1.3. There is a dual picture in which bubbles replace corollas, marked points replace leaves, and the root is denoted as a point labeled $\infty$ (Figure 1 $1 \mathrm{~b}$ ). Using the above notation, the composition $s \circ_{i} t$ is defined by fusing the $\infty$ of the bubble $s$ with the $i^{t h}$ marked point of $t$. The branches of the tree are now identified with double points, the places where bubbles intersect.

\footnotetext{
${ }^{1} \mathrm{~A}$ corolla is a collection of edges meeting at a common vertex.
} 

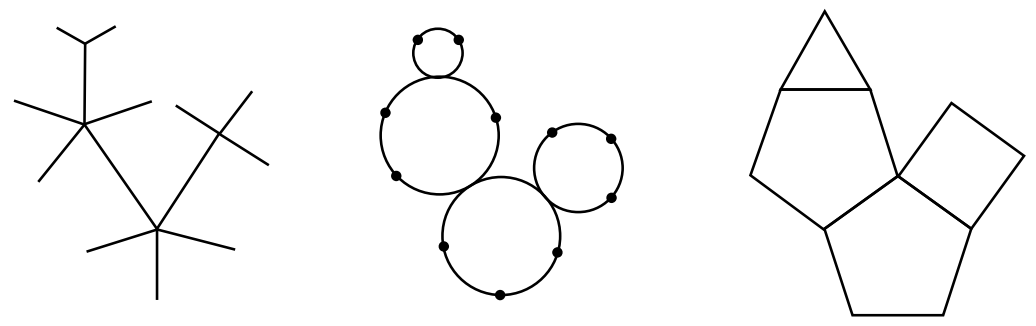

Figure 1. Trees, Bubbles, and Polygons

1.2. Taking yet another dual, we can define an operad structure on a collection of polygons (modulo an appropriate equivalence relation) as shown in Figure 11c. Each bubble corresponds to a polygon, where the number of marked and double points become the number of sides; the fusing of points is associated with the gluing of faces. The nicest feature of polygons is that, unlike corollas and bubbles, the iterated composition of polygons yields a polygon with marked diagonals (Figure 2).

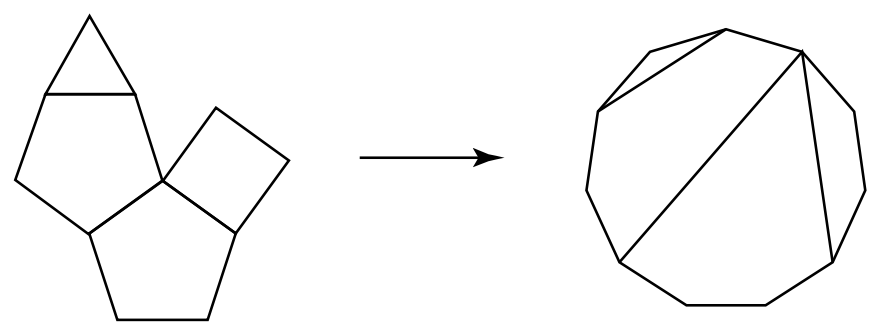

FiguRE 2.

Unlike the rooted trees, this mosaic operad is cyclic in the sense of Getzler and Kapranov [6, §2]. The most basic case (Figure 3) shows how two polygons, with sides labeled $a$ and $b$ respectively, compose to form a new polygon. The details of this operad are made precise in $\$ 3.1$.

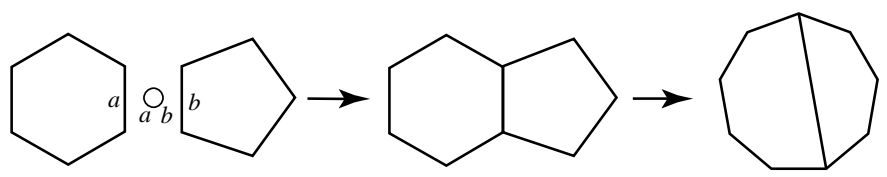

Figure 3. Polygon composition

1.3. In the work of Boardman and Vogt [2, §2.6], an operad is presented using $m$ dimensional cubes $I^{m} \subset \mathbb{R}^{m}$. An element $\mathcal{C}(n)$ of this little cubes operad is the space of an ordered collection of $n$ cubes linearly embedded by $f_{i}: I^{m} \hookrightarrow I^{m}$, with disjoint interiors and axes parallel to $I^{m}$. The $f_{i}$ 's are uniquely determined by the $2 n$-tuple of points $\left(a_{1}, b_{1}, \ldots, a_{n}, b_{n}\right)$ in $I^{m}$, corresponding to the images of the lower and upper vertices of $I^{m}$. An element $\sigma \in \mathbb{S}_{n}$ acts on $\mathcal{C}(n)$ by permuting the labeling of each cube:

$$
\left(a_{1}, b_{1}, \ldots, a_{n}, b_{n}\right) \mapsto\left(a_{\sigma(1)}, b_{\sigma(1)}, \ldots, a_{\sigma(n)}, b_{\sigma(n)}\right) .
$$



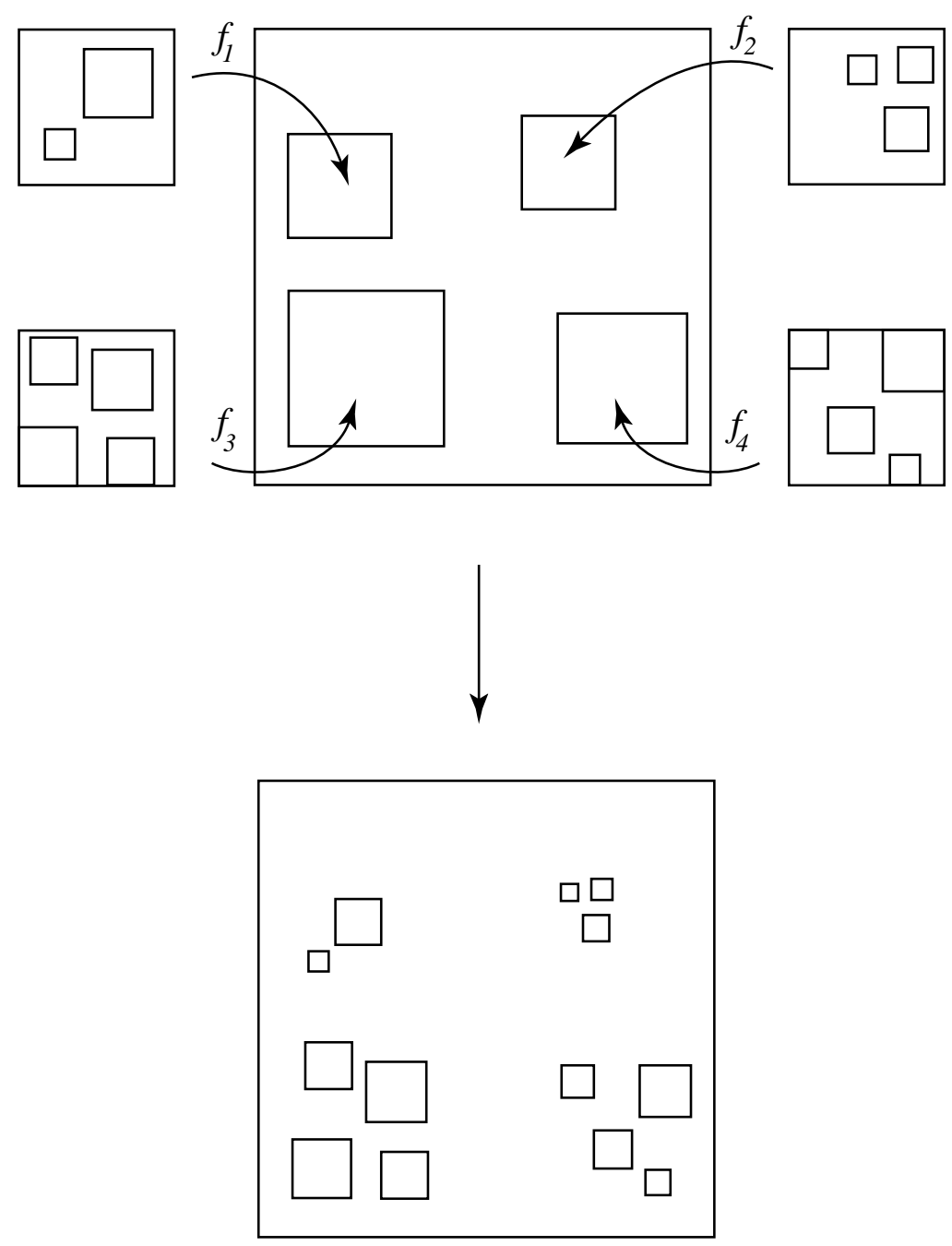

Figure 4. Little cubes composition

The composition operation (1.1) is defined by taking $n$ spaces $\mathcal{C}\left(k_{i}\right)$ (each having $k_{i}$ embedded cubes) and embedding them as an ordered collection into $\mathfrak{C}(n)$. Figure 4 shows an example for the two dimensional case when $n=4$.

Boardman showed that the space of $n$ distinct cubes in $\mathbb{R}^{m}$ is homotopically equivalent to $\operatorname{Config}^{n}\left(\mathbb{R}^{m}\right)$, the configuration space on $n$ distinct points in $\mathbb{R}^{m}$. When $m=2$, $\operatorname{Config}^{n}\left(\mathbb{R}^{2}\right)$ is homeomorphic to $\mathbb{C}^{n}-\Delta$, where $\Delta$ is the thick diagonal $\left\{\left(x_{1}, \ldots, x_{n}\right) \in \mathbb{C}^{n} \mid \exists i, j, i \neq j, x_{i}=x_{j}\right\}$. Since the action of $\mathbb{S}_{n}$ on $\mathbb{C}^{n}-\Delta$ is free, taking the quotient yields another space $\left(\mathbb{C}^{n}-\Delta\right) / \mathbb{S}_{n}$. It is wellknown that both these spaces are aspherical, having all higher homotopy groups vanish [4]. The following short exact sequence of fundamental groups results:

$$
\pi_{1}\left(\mathbb{C}^{n}-\Delta\right) \longmapsto \pi_{1}\left(\left(\mathbb{C}^{n}-\Delta\right) / \mathbb{S}_{n}\right) \rightarrow \mathbb{S}_{n}
$$

\footnotetext{
${ }^{2}$ The equivariant version of this theorem is proved by May in 13 , §4].
} 
But $\pi_{1}$ of $\mathbb{C}^{n}-\Delta$ is simply $\mathbf{P}_{n}$, the pure braid group. Similarly, $\pi_{1}$ of $\mathbb{C}^{n}-\Delta$ quotiented by all permutations of labelings is the braid group $\mathbf{B}_{n}$. Therefore, the short exact sequence above takes on the more familiar form:

$$
\mathbf{P}_{n} \longmapsto \mathbf{B}_{n} \rightarrow \mathbb{S}_{n}
$$

We will return to these ideas in $\S 6$.

\section{The Moduli Space}

2.1. The moduli space of Riemann spheres with $n$ punctures,

$$
\mathcal{M}_{0}^{n}(\mathbb{C})=\left(\operatorname{Config}^{n}\left(\mathbb{C P}^{1}\right)\right) / \mathbb{P G l}_{2}(\mathbb{C})
$$

has been studied extensively 10]. It has a Deligne-Mumford compactification $\overline{\mathcal{M}}_{0}^{n}(\mathbb{C})$, a smooth variety of complex dimension $n-3$. In fact, this variety is defined over the integers; we will look at the real points of this space.

Definition 2.1.1. The moduli space $\overline{\mathcal{M}}_{0}^{n}(\mathbb{R})$ of configurations of $n$ smooth points on punctured stable real algebraic curves of genus zero is a compactification of the quotient $\left.\left((\mathbb{R P})^{1}\right)^{n}-\Delta\right) / \mathbb{P G l}_{2}(\mathbb{R})$, where $\Delta$ is the thick diagonal.

Remark. This is an action of a non-compact group on a non-compact space. Geometric invariant theory gives a natural compactification for this quotient, defined combinatorially in terms of bubble trees or algebraically as a moduli space of real algebraic curves of genus zero with $n$ points, which are stable in the sense that they have only finitely many automorphisms.

A point of $\mathcal{M}_{0}^{n}(\mathbb{R})$ can be visualized as a bubble (that is, $\mathbb{R P}^{1}$ ) with $n$ distinct marked points. In $\overline{\mathcal{M}}_{0}^{n}(\mathbb{R})$, however, these marked points are allowed to 'collide' in the following sense: As two adjacent points $p_{1}$ and $p_{2}$ of the bubble come closer together and try to collide, the result is a new bubble fused to the old at the point of collision (a double point), where the marked points $p_{1}$ and $p_{2}$ are now on the new bubble (Figure 5). Note that each bubble must have at least three marked or double points in order to be stable.
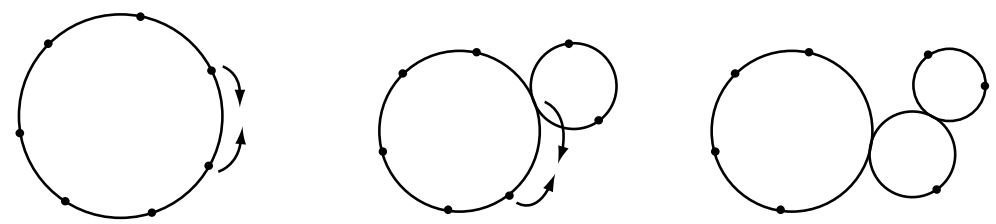

FIGURE 5 .

The mosaic operad encapsulates all the information of the bubbles, enabling one to look at the situation above from the vantage point of polygons. Having $n$ marked points on a circle now corresponds to an $n$-gon; when two adjacent sides $p_{1}$ and $p_{2}$ of the polygon try to collide, a diagonal of the polygon is formed such that $p_{1}$ and $p_{2}$ lie on one side of the diagonal (Figure 6). This can be generalized: A place where $k+1$ marked points on $\mathbb{R P}^{1}$ collide corresponds to having a diagonal $d$ partition an $n$-gon in such a way that $k+1$ points lie on one side of $d$ and $n-k-1$ points lie on the other. Therefore, the cells of $\overline{\mathcal{M}}_{0}^{n}(\mathbb{R})$ correspond to polygons with diagonals. The exact formulation of this appears in $\S$. 

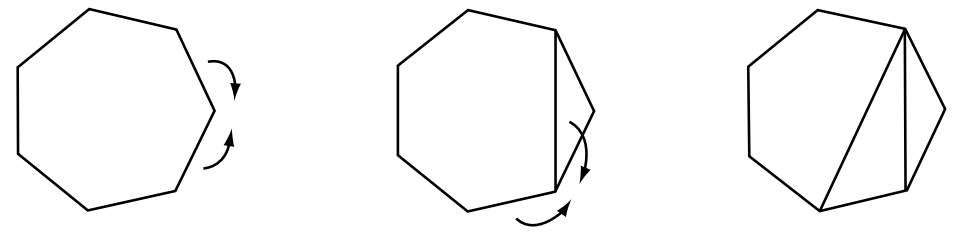

FiguRE 6.

2.2. There are two elegant approaches in better understanding this space. One is to view $\overline{\mathcal{M}}_{0}^{n}(\mathbb{R})$ in terms of hyperplane arrangements as formulated by Kapranov [8, §4.3] and described by Davis, Januszkiewicz, and Scott [5, §0.1].

Definition 2.2.1. Let $V^{n} \subset \mathbb{R}^{n-1}$ be the hyperplane defined by $\Sigma x_{i}=0$. For $1 \leq i<j \leq n-1$, let $H_{i j}^{n} \subset V^{n}$ be the hyperplane defined by $x_{i}=x_{j}$. The braid arrangement is the collection of subspaces of $V^{n}$ generated by all possible intersections of the $H_{i j}^{n}$.

Notation. If $\mathcal{H}^{n}$ denotes the collection of subspaces $H_{i j}^{n}$, then $\mathcal{H}^{n}$ cuts $V^{n}$ into simplicial cones. Let $\mathbb{P}\left(V^{n}\right)$ be the projective sphere in $V^{n}$, that is, $\mathbb{R P} \mathbb{P}^{n-3}$. Let $\mathcal{B}^{n}$ be the intersection of $\mathcal{H}^{n}$ with $\mathbb{P}\left(V^{n}\right)$ and let $\mathfrak{b}^{k}$ be a $k$-dimensional irreducible component of $\mathcal{B}^{n}$. The arrangement $\mathcal{B}^{n}$ cuts $\mathbb{P}\left(V^{n}\right)$ into open $(n-3)$-simplicies; the irreducible components $\left\{\mathfrak{b}^{k}\right\}$ comprise the boundaries of these simplicies.

Definition 2.2.2. Replace $\mathfrak{b}^{k}$ with $\mathfrak{s b}^{k}$, the sphere bundle associated to the normal bundle of $\mathfrak{b}^{k} \subset \mathbb{P}\left(V^{n}\right)$. This process yields a manifold with boundary. Then projectify $\mathfrak{s b}^{k}$ into $\mathfrak{p b}^{k}$, the projective sphere bundle. This defines a manifold without boundary, called the blow-up of $\mathbb{P}\left(V^{n}\right)$ along $\mathfrak{b}^{k}$.

Remark. Replacing $\mathfrak{b}^{k}$ with $\mathfrak{s b}^{k}$ for any dimension $k$ creates a new manifold with boundary. However, blowing up along $\mathfrak{b}^{k}$ defines a new manifold for all dimensions except codim one. That is, for codim one, projectifying $\mathfrak{s b}^{k}$ into $\mathfrak{p b}^{k}$ annuls the process of replacing $\mathfrak{b}^{k}$ with $\mathfrak{s b}^{k}$.

Proposition 2.2.3. [8, §4.3] The iterated blow-up of $\mathbb{P}\left(V^{n}\right)$ along the cells $\left\{\mathfrak{b}^{k}\right\}$ in increasing order of dimension yields $\overline{\mathcal{M}}_{0}^{n}(\mathbb{R})$.

Therefore, the compactification of $\mathcal{M}_{0}^{n}(\mathbb{R})$ is obtained by replacing the set $\left\{\mathfrak{b}^{k}\right\}$ with $\left\{\mathfrak{p b}^{k}\right\}$. The closure of $\mathcal{M}_{0}^{n}(\mathbb{R})$ in $\mathbb{P}\left(V^{n}\right)$, denoted by $\widehat{\mathcal{M}}_{0}^{n}(\mathbb{R})$, is obtained by replacing the set $\left\{\mathfrak{b}^{k}\right\}$ with $\left\{\mathfrak{s b}^{k}\right\}$; this procedure truncates each simplex of $\mathbb{P}\left(V^{n}\right)$ into an $n-3$ dimensional polytope. The resulting polytope after truncation is called the associahedron $K_{n-1}$. Each $K_{n-1}$ is naturally given a Riemann metric coming from $\mathcal{M}_{0}^{n}(\mathbb{R})$. The properties of the associahedra are examined in $\$$.

Historical Note. Stasheff originally defined the associahedra in a purely combinatorial manner for use in homotopy theory [17, §6]. Since then, they have continued to appear in a vast number of mathematical fields, gradually acquiring more and more structure, $c f$. [19.

\footnotetext{
${ }^{3}$ It is inessential to specify the order in which cells $\left\{\mathfrak{b}^{k}\right\}$ of the same dimension are blown up.

${ }^{4}$ For a detailed construction of this truncation, see Appendix B of [16].
} 
Example 2.2.4. The diagram of $\overline{\mathcal{M}}_{0}^{5}(\mathbb{R})$ is shown in Figure 7, first found in a different context by Brahana and Coble in 1926 [1, §1]. The arrangement $\mathcal{B}^{5}$ on $\mathbb{P}\left(V^{5}\right) \simeq \mathbb{R} \mathbb{P}^{2}$ yields six lines forming twelve 2-simplicies; the irreducible components of $\operatorname{dim} 0$ turn out to be the points $\left\{\mathfrak{b}_{1}^{0}, \ldots, \mathfrak{b}_{4}^{0}\right\}$ of triple intersection. Blowing up along these components, we get $S^{1}$ as a hexagon for $\mathfrak{s b}_{i}^{0}$ and $\mathbb{R} \mathbb{P}^{1}$ as a triangle for $\mathfrak{p b}_{i}^{0}$. The associahedron $K_{4}$ is seen to be a pentagon, and the space $\overline{\mathcal{M}}_{0}^{5}(\mathbb{R})$ becomes tessellated by twelve such cells (shaded), a somewhat "evil twin" of the dodecahedron.

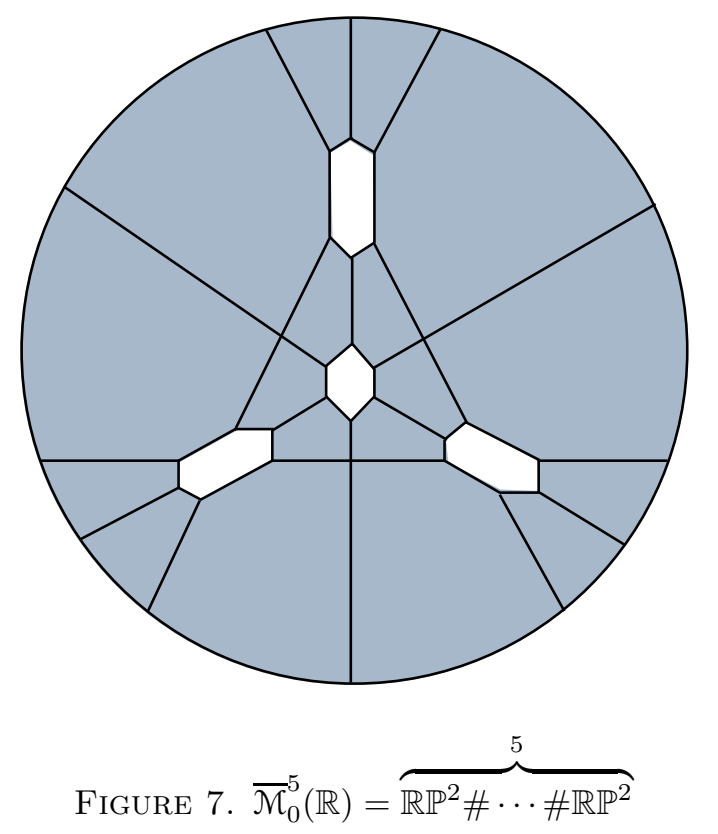

2.3. Another way of looking at the moduli space comes from observing the inclusion $\mathbb{S}_{3} \subset \mathbb{P G l} l_{2}(\mathbb{R})$. Since $\overline{\mathcal{M}}_{0}^{n}(\mathbb{R})$ is defined as $n$ distinct points on $\mathbb{R P}^{1}$ quotiented by $\mathbb{P G l}_{2}(\mathbb{R})$, one can fix three of these points to be 0,1 , and $\infty$. From this perspective we see that $\overline{\mathcal{M}}_{0}^{3}(\mathbb{R})$ is a point. When $n=4$, the cross-ratio is a homeomorphism from $\overline{\mathcal{M}}_{0}^{4}(\mathbb{R})$ to $\mathbb{R P}^{1}$, the result of identifying three of the four points with 0,1 , and $\infty$. In general, $\overline{\mathcal{M}}_{0}^{n}(\mathbb{R})$ becomes a manifold blown up from an $n-3$ dimensional torus, coming from the $n-3$-fold products of $\mathbb{R P}^{1}$. Therefore, the moduli space before compactification can be defined as

$$
\left(\left(\mathbb{R P}^{1}\right)^{n}-\Delta^{*}\right) / \mathbb{P G l} 2(\mathbb{R}),
$$

where $\Delta^{*}=\left\{\left(x_{1}, \ldots, x_{n}\right) \in\left(\mathbb{R} \mathbb{P}^{1}\right)^{n} \mid\right.$ at least 3 points collide $\}$.

Proposition 2.3.1. [18, §5.3] $\mathcal{M}_{0}^{n}(\mathbb{R})$ is compactified by blowing up along $\Delta^{*}$.

Remark. For $0 \leq k \leq n-5, \Delta^{*}$ coincides exactly with $\left\{\mathfrak{b}^{k}\right\} \subset \mathcal{B}^{n}$. Therefore, places where $k+1$ points collide correspond to codim $k$ cells $\mathfrak{b}_{i}^{n-3-k}$. 


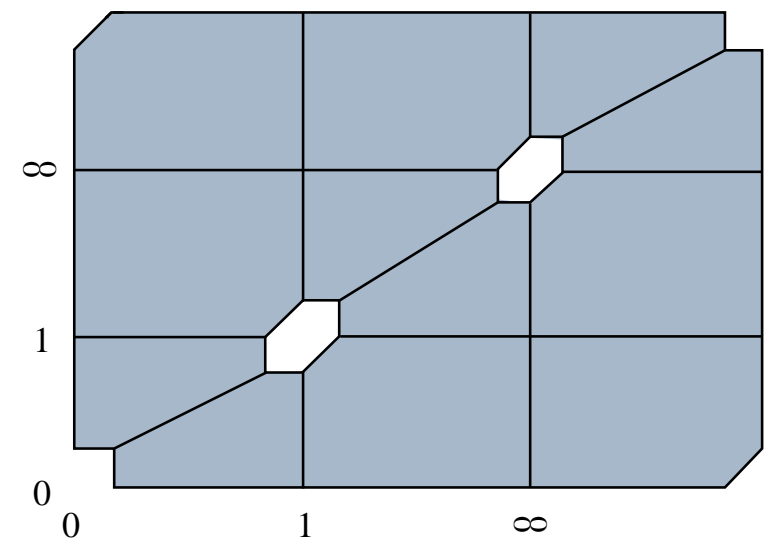

Figure 8. $\overline{\mathcal{M}}_{0}^{5}(\mathbb{R})=\mathbb{T}^{2} \# \overbrace{\mathbb{R P}^{2} \# \cdots \# \mathbb{R P}^{2}}^{3}$

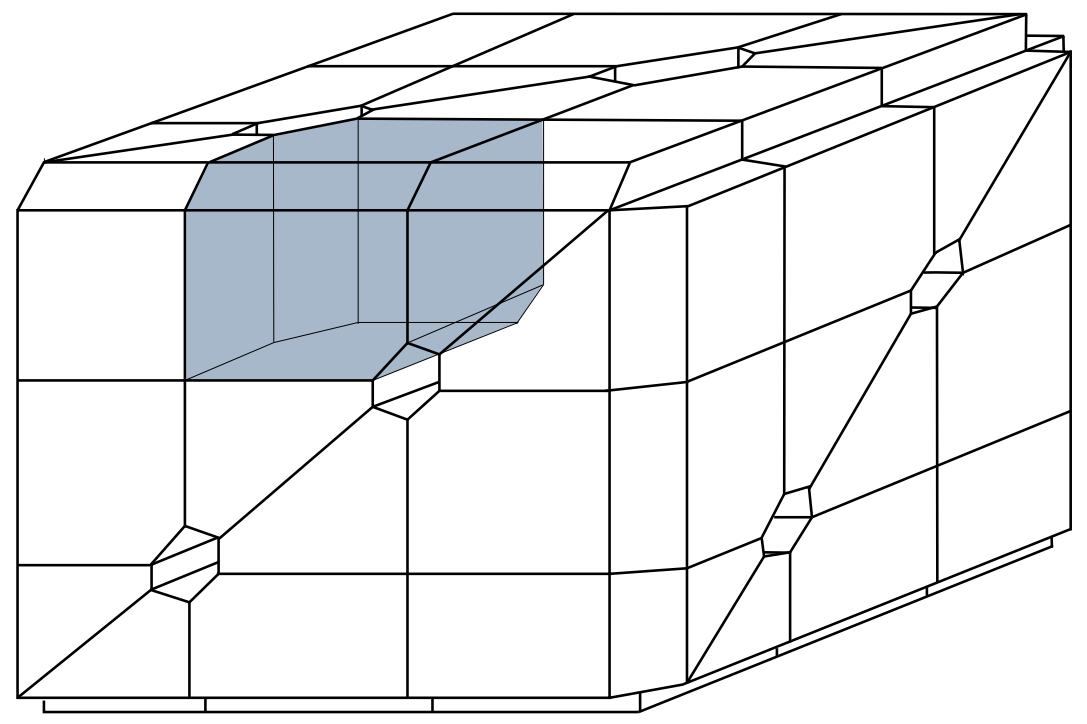

Figure $9 . \overline{\mathcal{M}}_{0}^{6}(\mathbb{R})$

Example 2.3.2. An illustration of $\overline{\mathcal{M}}_{0}^{5}(\mathbb{R})$ from this perspective appears in Figure 8 . From the five marked points on $\mathbb{R P}^{1}$, three are fixed leaving two dimensions to vary, say $x_{1}$ and $x_{2}$. The set $\Delta$ is made up of seven lines $\left\{x_{1}, x_{2}=0,1, \infty\right\}$ and $\left\{x_{1}=x_{2}\right\}$, giving a space tessellated by six squares and six triangles. Furthermore, $\Delta^{*}$ becomes the set of three points $\left\{x_{1}=x_{2}=0,1, \infty\right\}$; blowing up along these points yields the space $\overline{\mathcal{M}}_{0}^{5}(\mathbb{R})$ tessellated by twelve pentagons.

Example 2.3.3. In Figure 9, a rough sketch of $\overline{\mathcal{M}}_{0}^{6}(\mathbb{R})$ is shown as the blow-up of a three torus. The set $\Delta^{*}$ associated to $\overline{\mathcal{M}}_{0}^{6}(\mathbb{R})$ has ten lines $\left\{x_{i}=x_{j}=0,1, \infty\right\}$ and $\left\{x_{1}=x_{2}=x_{3}\right\}$, and three points $\left\{x_{1}=x_{2}=x_{3}=0,1, \infty\right\}$. The lines correspond to the hexagonal prisms, nine cutting through the faces, and the tenth (hidden) 
running through the torus from the bottom left to the top right corner. The three points correspond to places where four of the prisms intersect.

The shaded region has three squares and six pentagons as its codim one faces. In fact, all the top dimensional cells that form $\overline{\mathcal{M}}_{0}^{6}(\mathbb{R})$ turn out to have this property; these cells are the associahedra $K_{5}$.

\section{The Associahedron}

3.1. We now turn to defining the mosaic operad and relating its properties with the cellular structure of the associahedron. Let $S^{1}$ be the unit circle bounding $\mathfrak{D}$, the conformal disk endowed with the Poincaré metric. The geodesics in $\mathfrak{D}$ correspond to open diameters of $S^{1}$ together with open circular arcs orthogonal to $S^{1}$. The group of isometries on $\mathfrak{D}$ is $\mathbb{P G l}_{2}(\mathbb{R})[15, \S 4]$.

Associate $S^{1}$ with $\mathbb{R} \mathbb{P}^{1}$. Between two adjacent points of an element in $\operatorname{Config}^{n}\left(\mathbb{R} \mathbb{P}^{1}\right)$, draw a geodesic in $\mathfrak{D}$. Let $\widetilde{\mathcal{G}}(n, 0)$ be the space of such geodesics coming from $\operatorname{Config}^{n}\left(\mathbb{R} \mathbb{P}^{1}\right)$. In general, let $\widetilde{\mathcal{G}}(n, k)$ be the space $\widetilde{\mathcal{G}}(n, 0)$ with $k$ non-intersecting geodesics between non-adjacent points. Define the space $\mathcal{G}(n, k)$ to be $\widetilde{\mathcal{G}}(n, k) / \mathbb{P G l}(\mathbb{R})$. Henceforth, we will visualize an element of $\mathcal{G}(n, k)$ as an $n$-gon with $k$ non-intersecting diagonals (Figure 10). For an arbitrary number of diagonals, we write $\mathcal{G}(n)$.

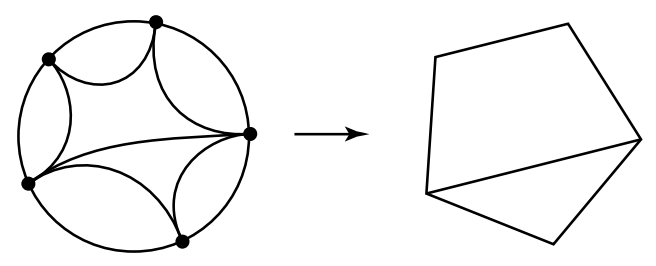

FigURE 10.

Definition 3.1.1. Given $G \in \mathcal{G}(m, l)$ and $G_{i} \in \mathcal{G}\left(n_{i}, k_{i}\right)$ (where $1 \leq i \leq m$ ), there are composition maps

$$
G_{a_{1} \circ b_{1}} G_{1} a_{2} \circ_{b_{2}} \cdots a_{a_{m}} \circ_{b_{m}} G_{m} \mapsto G_{t}
$$

where $G_{t} \in \mathcal{G}\left(-m+\sum n_{i}, m+l+\sum k_{i}\right)$. The object $G_{t}$ is obtained by gluing side $a_{i}$ of $G$ along side $b_{i}$ of $G_{i}$. The symmetric group $\mathbb{S}_{n}$ acts on $G_{n}$ by permuting the labeling of the sides. These operations define the mosaic operad $\{\mathcal{G}(n, k)\}$.

Remark. The one dimensional case of the little cubes operad is $\{\mathrm{J}(n)\}$, the little intervals operad. An element $\mathcal{J}(n)$ is defined to be an ordered collection of $n$ embeddings of the interval $I \hookrightarrow I$, with disjoint interiors. The notion of trees and bubbles, shown in Figure 1, is encapsulated in this intervals operad. Furthermore, after embedding $I$ in $\mathbb{R}$ and identifying $\mathbb{R} \cup \infty$ with $\mathbb{R P}^{1}$, we note that the mosaic operad $\{\mathcal{G}(n, k)\}$ is a compactification of $\{\mathcal{J}(n)\}$.

3.2. We now define the associahedron $K_{n-1}$ as a concrete geometric object and present its connections with the mosaic operad.

Definition 3.2.1. Let $\mathcal{A}$ be the space of $n-3$ distinct points $\left\{t_{1}, \ldots, t_{n-3}\right\}$ on the interval $[0,1]$ such that $0<t_{1}<\cdots<t_{n-3}<1$. Identifying $\mathbb{R} \cup \infty$ with $\mathbb{R P}^{1}$ carries the set $\left\{0, t_{1}, \ldots, t_{n-3}, 1, \infty\right\}$ of $n$ points onto $\mathbb{R} \mathbb{P}^{1}$ (Figure 11). Therefore, 
there exists a natural inclusion of $\mathcal{A}$ in $\overline{\mathcal{M}}_{0}^{n}(\mathbb{R})$. The associahedron $K_{n-1}$ can be defined as the closure of the space $\mathcal{A}$ in $\overline{\mathcal{M}}_{0}^{n}(\mathbb{R})$.
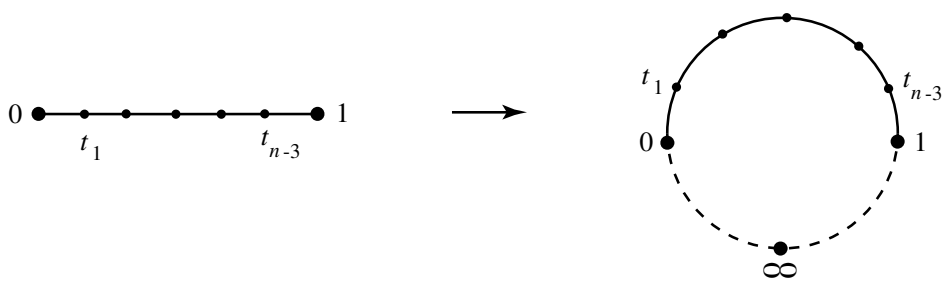

Figure 11.

Define $\mathcal{G}^{L}(n, k)$ to be the space $\mathcal{G}(n, k)$ with the geodesic sides labeled with all permutations of $\{1, \ldots, n\}$. The following is an immediate consequence of the discussion in 2.1 and the construction of $\mathcal{G}(n, k)$.

Proposition 3.2.2. There exists a bijection between the points of $\widehat{\mathcal{M}}_{0}^{n}(\mathbb{R})$ and the elements of $\mathcal{G}^{L}(n, k)$.

Since the associahedron $K_{n-1}$ can be identified with the cell tiling the space $\widehat{\mathcal{M}}_{0}^{n}(\mathbb{R})$, it can be realized as a purely combinatorial object from its CW-complex structure. This results in

Proposition 3.2.3. An interior point of $K_{n-1}$ corresponds to an element of $\mathcal{G}(n, 0)$, and an interior point of a codim $k$ face corresponds to an element of $\mathcal{G}(n, k)$.

The relation between the $n$-gon and $K_{n-1}$ is further highlighted by a work of Lee [12], where he constructs a polytope $Q_{n}$ that is dual to $K_{n-1}$, with one vertex for each diagonal and one facet for each triangulation of an $n$-gon. He then proves the symmetry group of $Q_{n}$ to be the dihedral group $D_{n}$. Restated, it becomes

Proposition 3.2.4. [12, §5] The symmetry group of $K_{n-1}$ is $D_{n}$.

Remark. Stasheff classically defined $K_{n-1}$ as a CW-ball with codim $k$ faces corresponding to using $k$ sets of parentheses meaningfully on $n-1$ letters. . Later the associahedron was given a realization as an $n-3$ dimensional convex polytope by Milnor (unpublished). The two descriptions of the associahedron, using polygons and parentheses, are compatible: Figure 12 illustrates $K_{2}$ (point), $K_{3}$ (line), and $K_{4}$ (pentagon).

3.3. The polygon relation to the associahedron enables the use of the mosaic operad structure on $K_{n-1}$. The following brings in the operad's role in terms of decomposition.

Proposition 3.3.1. [17, §2] Each face of $K_{n-1}$ is a product of lower dimensional associahedra.

In general, the codim $k-1$ face of the associahedron $K_{m-1}$ will decompose as

$$
K_{n_{1}-1} \times \cdots \times K_{n_{k}-1} \hookrightarrow K_{m-1},
$$

\footnotetext{
${ }^{5}$ From the definition above, the $n-1$ letters can be viewed as the points $\left\{0, t_{1}, \ldots, t_{n-3}, 1\right\}$.
} 

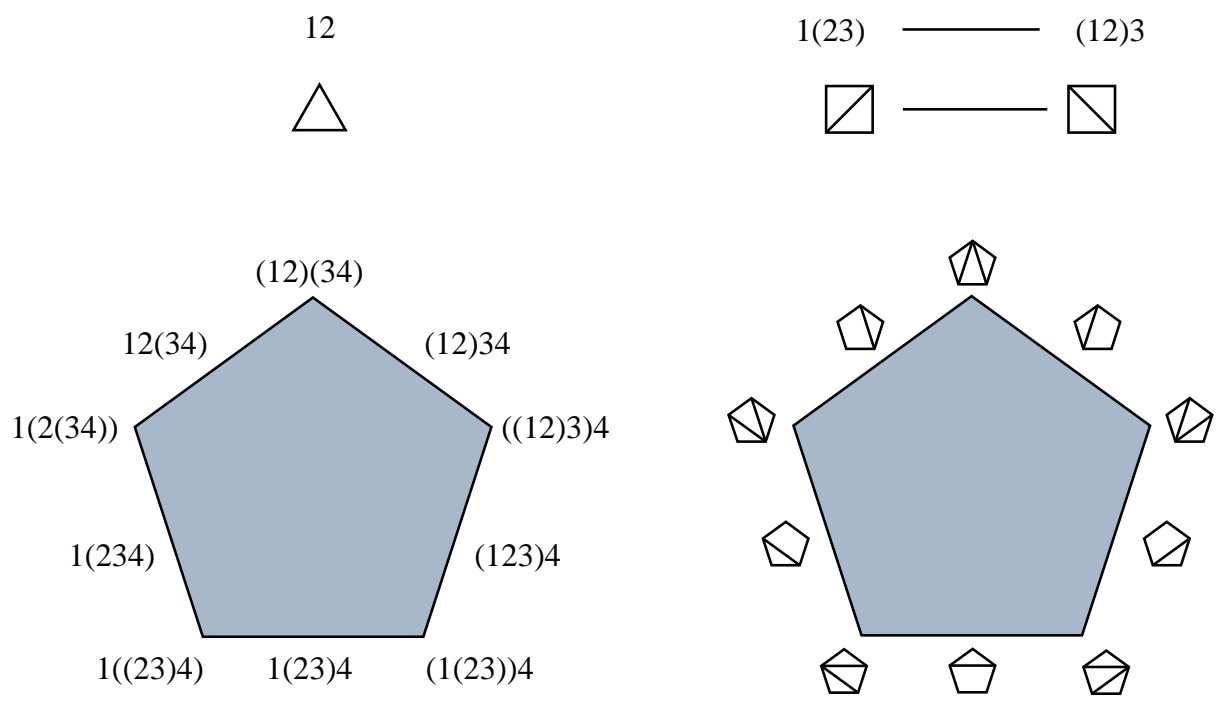

Figure 12. Associahedra $K_{2}, K_{3}$, and $K_{4}$

where $\sum n_{i}=m+2(k-1)$ and $n_{i} \geq 3$. This parallels the mosaic operad structure

$$
G\left(n_{1}\right) \circ \cdots \circ G\left(n_{k}\right) \mapsto G(m),
$$

where $G\left(n_{i}\right) \in \mathcal{G}\left(n_{i}, 0\right), G(m) \in \mathcal{G}(m, k-1)$, and the gluing of sides is arbitrary. Therefore, the product in Proposition 3.3.1 is indexed by the internal vertices of the tree corresponding to the face of the associahedron.

Example 3.3.2. We look at the codim one faces of $K_{5}$. The three dimensional $K_{5}$ corresponds to a 6 -gon, which has two distinct ways of adding a diagonal. One way, in Figure 13 a, will allow the 6-gon to decompose into a product of two 4-gons ( $K_{3}$ 's). Since $K_{3}$ is a line, this codim one face yields a square. The other way, in Figure $13 \mathrm{~b}$, decomposes the 6 -gon into a 3 -gon $\left(K_{2}\right)$ and a 5 -gon $\left(K_{4}\right)$. Taking the product of a point and a pentagon results in a pentagon. Note how this description of $K_{5}$ matches the shaded region in Figure 9 .

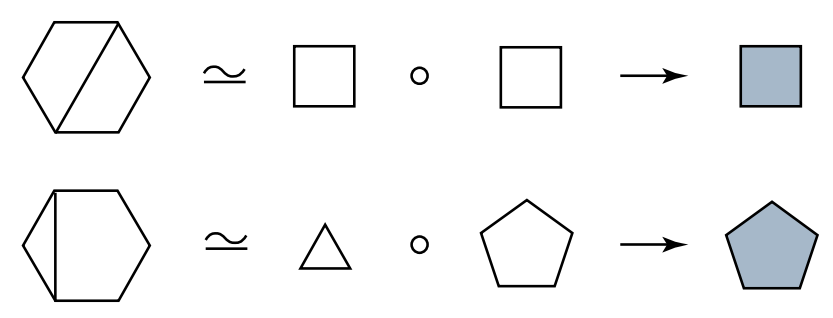

Figure 13. Codim one cells of $K_{5}$

Example 3.3.3. We look at the codim one faces of $K_{6}$. Similar to the ideas above, Figure 14 shows the decomposition of the two types of codim one faces of $K_{6}$, a pentagonal prism and $K_{5}$. 


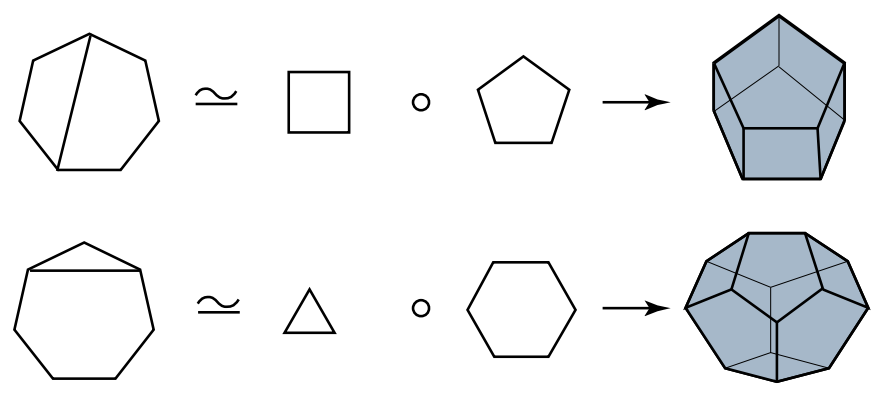

Figure 14. Codim one cells of $K_{6}$

\section{The Tessellation}

4.1. We extend the combinatorial structure of the associahedra to $\overline{\mathcal{M}}_{0}^{n}(\mathbb{R})$. Propositions 3.2 .2 and 3.2 .3 show the correspondence between the associahedra in $\widehat{\mathcal{M}}_{0}^{n}(\mathbb{R})$ and $\mathcal{G}^{L}(n, k)$. We investigate how these copies of $K_{n-1}$ glue to form $\overline{\mathcal{M}}_{0}^{n}(\mathbb{R})$.

Definition 4.1.1. Let $G \in \mathcal{G}^{L}(n, k)$ and $d$ be a diagonal of $G$. A twist along $d$, denoted by $\nabla_{d}(G)$, is the element of $\mathcal{G}^{L}(n, k)$ obtained by 'breaking' $G$ along $d$ into two parts, reflecting (or 'twisting') one of the pieces, and 'gluing' them back together (Figure 15).
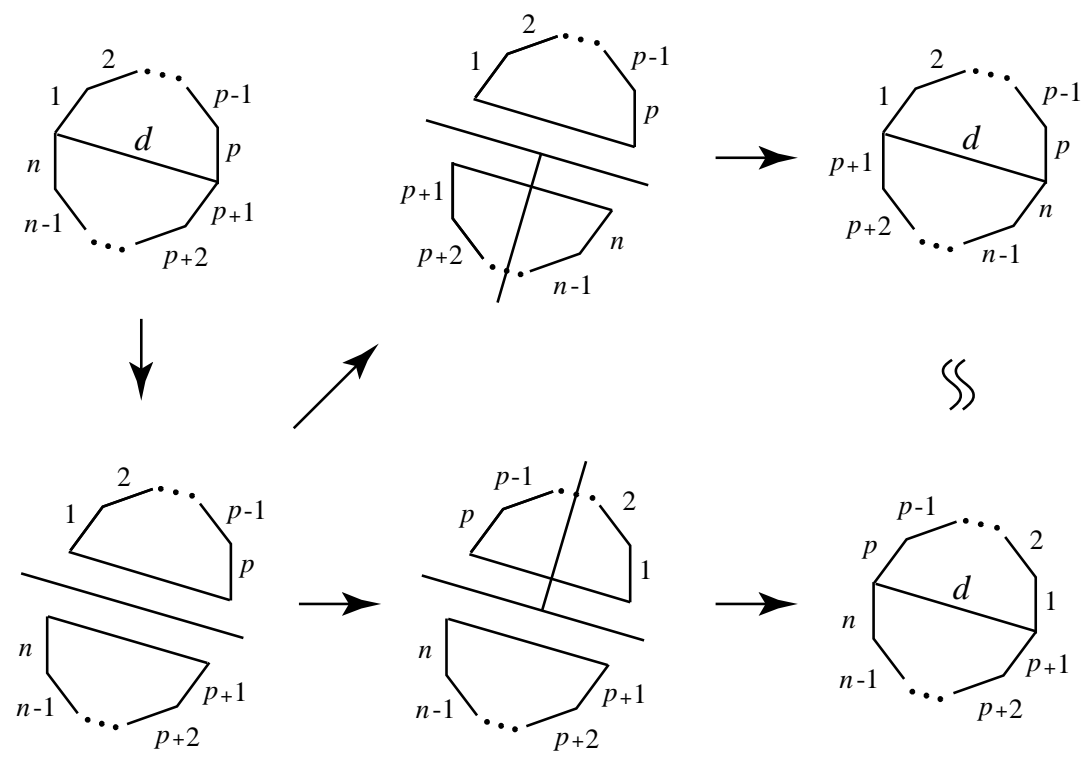

Figure 15. Twist along $d$

The twisting operation is well-defined since the diagonals of an element in $\mathcal{G}^{L}(n, k)$ do not intersect. Furthermore, it does not matter which piece of the polygon is twisted since the two results are identified by an action of $D_{n}$. It follows immediately that $\nabla_{d} \cdot \nabla_{d}=e$, the identity element. 


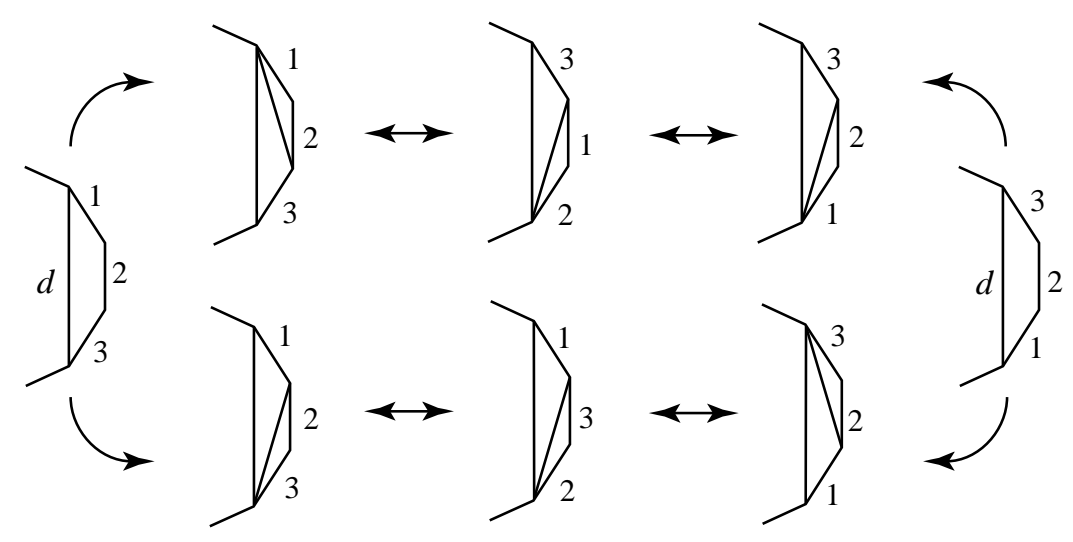

Figure 16.

Proposition 4.1.2. Two elements, $G_{1}, G_{2} \in \mathcal{G}^{L}(n, k)$, representing codim $k$ faces of associahedra, are identified in $\overline{\mathcal{M}}_{0}^{n}(\mathbb{R})$ if there exist diagonals $d_{1}, \ldots, d_{r}$ of $G_{1}$ such that

$$
\left(\nabla_{d_{1}} \cdots \nabla_{d_{r}}\right)\left(G_{1}\right)=G_{2}
$$

Proof. As two adjacent points $p_{1}$ and $p_{2}$ on $\mathbb{R P}^{1}$ collide, the result is a new bubble fused to the old at a point of collision $p_{3}$, where $p_{1}$ and $p_{2}$ are on the new bubble (see $\$ 2.1$ ). The location of the three points $p_{i}$ on the new bubble is irrelevant since $\mathbb{S}_{3} \subset \mathbb{P G l}_{2}(\mathbb{R})$. In the language of polygons, this means $\nabla_{d}$ does not affect the cell, where $d$ is the diagonal representing the double point $p_{3}$. In general, it follows that the labels of triangles can be permuted without affecting the cell. Let $G$ be an $n$-gon and let $d$ be a diagonal partitioning $G$ into a square and an $(n-2)$-gon. Figure 16 shows that since the square decomposes into triangles, the cell corresponding to $G$ is invariant under the action of $\nabla_{d}$. Since any partition of $G$ by a diagonal $d$ can be decomposed into triangles, it follows by induction that $\nabla_{d}$ does not affect the cell corresponding to $G$.

Theorem 4.1.3. There exists a surjection

$$
K_{n-1} \times_{D_{n}} \mathbb{S}_{n} \rightarrow \overline{\mathcal{M}}_{0}^{n}(\mathbb{R})
$$

which is a bijection on the interior of the cells. In particular, $\frac{1}{2}(n-1)$ ! copies of $K_{n-1}$ tessellate $\overline{\mathrm{M}}_{0}^{n}(\mathbb{R})$.

Proof. Each $K_{n-1}$ in $\overline{\mathrm{M}}_{0}^{n}(\mathbb{R})$ is associated to a particular labeling on an $n$-gon (see $\S 3.2)$. Since there exists a copy of the dihedral group $D_{n}$ in $\mathbb{P G l}_{2}(\mathbb{R})$, and since $\overline{\mathcal{M}}_{0}^{n}(\mathbb{R})$ is defined as a quotient by $\mathbb{P G l}_{2}(\mathbb{R})$, then two labeled $n$-gons (corresponding to associahedra) are identified by an action of $D_{n}$. Therefore, there turn out to be $\frac{1}{2}(n-1)$ ! copies of $K_{n-1}$ that make up $\overline{\mathcal{M}}_{0}^{n}(\mathbb{R})$, with $n$ ! possible labelings coming from $\mathbb{S}_{n}$ and $2 n$ identifications coming from $D_{n}$. This gives the bijection above with the interior of the cells; the map is not an injection since the boundaries of the associahedra are glued according to Proposition 4.1.2.

4.2. In Figure 17, a piece of $\overline{\mathcal{M}}_{0}^{5}(\mathbb{R})$ represented by labeled polygons with diagonals is shown. Note how two codim one pieces (lines) glue together and four 


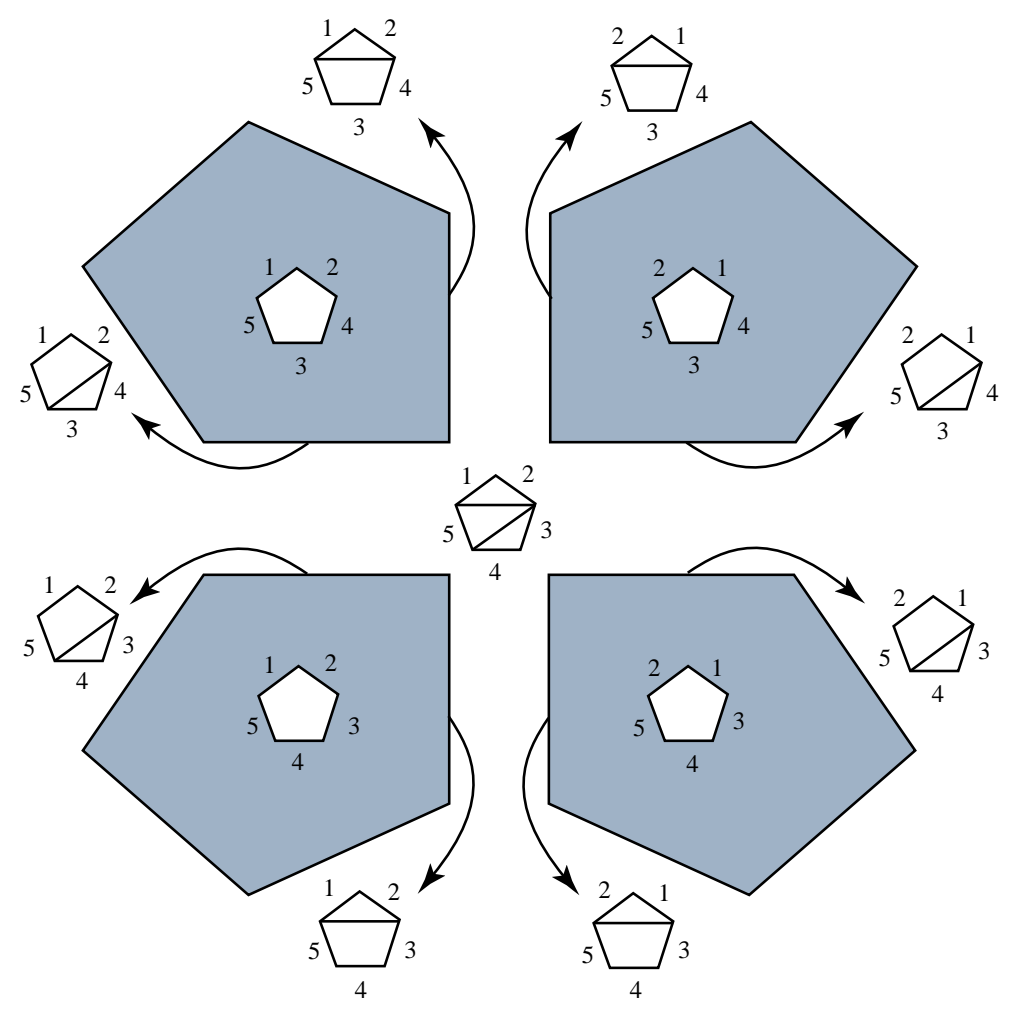

Figure 17. A piece of $\overline{\mathcal{M}}_{0}^{5}(\mathbb{R})$

codim two pieces (points) glue together. Understanding this gluing now becomes a combinatorial problem related to $\mathcal{G}^{L}(n, k)$.

Notation. Let $\Lambda(x, \mathfrak{X})$ be the number of codim $x$ cells in a CW-complex $\mathfrak{X}$. For a fixed codim $y_{2}$ cell in $\overline{\mathcal{M}}_{0}^{n}(\mathbb{R})$, and for $y_{1}<y_{2}$, let $\Lambda^{n}\left(y_{1}, y_{2}\right)$ be the number of codim $y_{1}$ cells in $\overline{\mathcal{M}}_{0}^{n}(\mathbb{R})$ whose boundary contains the codim $y_{2}$ cell. Note the number $\Lambda^{n}\left(y_{1}, y_{2}\right)$ is well-defined by Theorem 4.1.3.

Lemma 4.2.1.

$$
\Lambda\left(k, K_{n-1}\right)=\frac{1}{k+1}\left(\begin{array}{c}
n-3 \\
k
\end{array}\right)\left(\begin{array}{c}
n-1+k \\
k
\end{array}\right) .
$$

Proof. This is obtained by just counting the number of $n$-gons with $k$ non-intersecting diagonals, done by A. Cayley in 1891 [3].

\section{Lemma 4.2.2.}

$$
\Lambda^{n}(k-t, k)=2^{t}\left(\begin{array}{l}
k \\
t
\end{array}\right) .
$$

Proof. The boundary components of a cell corresponding to an element in $\mathcal{G}^{L}(n, k)$ are obtained by adding non-intersecting diagonals. To look at the coboundary cells, diagonals need to be removed. For each diagonal removed, two cells result (coming from the twist operation); removing $t$ diagonals gives $2^{t}$ cells. We then look at all possible ways of removing $t$ out of $k$ diagonals. 
Theorem 4.2.3.

$$
\chi\left(\overline{\mathcal{M}}_{0}^{n}(\mathbb{R})\right)= \begin{cases}0 & n \text { even } \\ (-1)^{\frac{n-3}{2}}(n-2)((n-4) ! !)^{2} & n \text { odd } .\end{cases}
$$

Proof. It is easy to show the following:

$$
\Lambda\left(k, \overline{\mathcal{M}}_{0}^{n}(\mathbb{R})\right) \cdot \Lambda^{n}(0, k)=\Lambda\left(0, \overline{\mathcal{M}}_{0}^{n}(\mathbb{R})\right) \cdot \Lambda\left(k, K_{n-1}\right) .
$$

Using Theorem 4.1.3 and Lemmas 4.2.1 and 1.2.2, we solve for $\Lambda\left(k, \overline{\mathcal{M}}_{0}^{n}(\mathbb{R})\right)$; but this is simply the number of codim $k$ cells in $\overline{\mathcal{M}}_{0}^{n}(\mathbb{R})$. Therefore,

$$
\chi\left(\overline{\mathcal{M}}_{0}^{n}(\mathbb{R})\right)=\sum_{k=0}^{n-3}(-1)^{n-3-k} \frac{(n-1) !}{2^{k+1}} \frac{1}{k+1}\left(\begin{array}{c}
n-3 \\
k
\end{array}\right)\left(\begin{array}{c}
n-1+k \\
k
\end{array}\right) .
$$

This equation can be reduced to the desired form.

Remark. Prof. F. Hirzebruch has kindly informed us that he has shown, using techniques of Kontsevich and Manin [11], that the signature of $\overline{\mathcal{M}}_{0}^{n}(\mathbb{C})$ is given by (4.1). He remarks that the equivalence of this signature with the Euler number of the space of real points is an elementary consequence of the Atiyah-Singer Gsignature theorem.

4.3. We now introduce a construction of Kapranov to give added clarity to $\overline{\mathcal{M}}_{0}^{n}(\mathbb{R})$.

Definition 4.3.1. [9, §4] A double cover of $\overline{\mathcal{M}}_{0}^{n}(\mathbb{R})$, denoted by $\widetilde{\mathcal{M}}_{0}^{n}(\mathbb{R})$, is obtained by fixing the $n^{\text {th }}$ marked point on $\mathbb{R} \mathbb{P}^{1}$ to be $\infty$ and assigning an orientation to this point.

Example 4.3.2. Figure 18 shows the polygon labelings of $\widetilde{\mathcal{M}}_{0}^{4}(\mathbb{R})$ and $\overline{\mathcal{M}}_{0}^{4}(\mathbb{R})$, being tiled by six and three copies of $K_{3}$ respectively. In this figure, the label 4 has been set to $\infty$. Note that the map $\widetilde{\mathcal{M}}_{0}^{4}(\mathbb{R}) \rightarrow \overline{\mathcal{M}}_{0}^{4}(\mathbb{R})$ is the antipodal quotient.

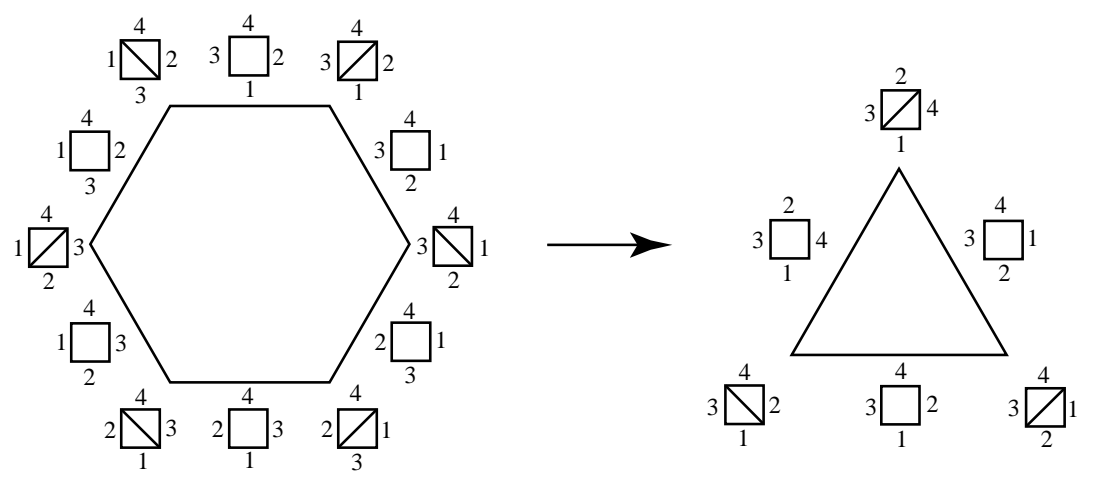

Figure $18 . \tilde{\mathcal{M}}_{0}^{4}(\mathbb{R}) \rightarrow \overline{\mathcal{M}}_{0}^{4}(\mathbb{R})$

The double cover can be constructed using blow-ups similar to the method described in $\$ 2.2$ for $\overline{\mathcal{M}}_{0}^{n}(\mathbb{R})$. However, instead of blowing up the projective sphere $\mathbb{P}\left(V^{n}\right)$, we blow-up $\mathbb{S}\left(V^{n}\right)$, the sphere in $V^{n}$. Therefore, except for the anomalous

${ }^{6}$ Kapranov uses the notation $\tilde{S}^{n-3}$ to represent this double cover. 
case of $\tilde{\mathcal{M}}_{0}^{4}(\mathbb{R})$, the double cover is a non-orientable manifold. Note also that the covering map $\widetilde{\mathcal{M}}_{0}^{n}(\mathbb{R}) \rightarrow \overline{\mathcal{M}}_{0}^{n}(\mathbb{R})$ is the antipodal quotient, coming from the map $\mathbb{S}\left(V^{n}\right) \rightarrow \mathbb{P}\left(V^{n}\right)$. Being a double cover, $\tilde{\mathcal{M}}_{0}^{n}(\mathbb{R})$ will be tiled by $(n-1)$ ! copies of $K_{n-1} \cdot 7$ It is natural to ask how these copies of $K_{n-1}$ will glue to form $\tilde{\mathcal{M}}_{0}^{n}(\mathbb{R})$.

Definition 4.3.3. A marked twist of an $n$-gon $G$ along its diagonal $d$, denoted by $\widetilde{\nabla}_{d}(G)$, is the polygon obtained by breaking $G$ along $d$ into two parts, reflecting the piece that does not contain the side labeled $\infty$, and gluing them back together.

The two polygons at the right of Figure 15 turn out to be different elements in $\tilde{\mathcal{M}}_{0}^{n}(\mathbb{R})$, whereas they are identified in $\overline{\mathcal{M}}_{0}^{n}(\mathbb{R})$ by an action of $D_{n}$. The following is an immediate consequence of the above definitions and Theorem 4.1.3.

Corollary 4.3.4 (of Theorem 4.1.3). There exists a surjection

$$
K_{n-1} \times_{\mathbb{Z}_{n}} \mathbb{S}_{n} \rightarrow \tilde{\mathcal{M}}_{0}^{n}(\mathbb{R})
$$

which is a bijection on the interior of the cells.

Remark. The spaces on the left define the classical $A_{\infty}$ operad [6, §2.9].

Theorem 4.3.5. There exists the following commutative diagram:

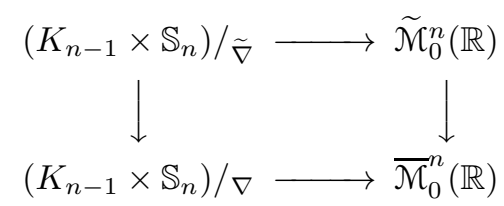

where the vertical maps are antipodal identifications and the horizontal maps are a quotient by $\mathbb{Z}_{n}$.

Proof. Look at $K_{n-1} \times \mathbb{S}_{n}$ by associating to each $K_{n-1}$ a particular labeling of an $n$-gon. We obtain $\left(K_{n-1} \times \mathbb{S}_{n}\right) / \widetilde{\nabla}$ by gluing the associahedra along codim one faces using $\widetilde{\nabla}$ (keeping the side labeled $\infty$ fixed). It follows that two associahedra will never glue if their corresponding $n$-gons have $\infty$ labeled on different sides of the polygon. This partitions $\mathbb{S}_{n}$ into $\mathbb{S}_{n-1} \cdot \mathbb{Z}_{n}$, with each element of $\mathbb{Z}_{n}$ corresponding to $\infty$ labeled on a particular side of the $n$-gon. Furthermore, Corollary 4.3.4 tells us that each set of the $(n-1)$ ! copies of $K_{n-1}$ glue to form $\tilde{\mathcal{M}}_{0}^{n}(\mathbb{R})$. Therefore, we get

$$
\left(K_{n-1} \times \mathbb{S}_{n}\right) / \tilde{\nabla}=\left(K_{n-1} \times \mathbb{S}_{n-1}\right) / \widetilde{\nabla} \times \mathbb{Z}_{n}=\tilde{\mathcal{M}}_{0}^{n}(\mathbb{R}) \times \mathbb{Z}_{n}
$$

\section{The Blow-Ups}

5.1. The only difference between $\overline{\mathcal{M}}_{0}^{n}(\mathbb{R})$ and $\mathbb{R P} \mathbb{P}^{n-3}$ is the blow-ups, making the study of their structures crucial. Looking at the arrangement $\mathcal{B}^{n}$ on $\mathbb{P}\left(V^{n}\right)$, there turn out to be $n-1$ irreducible $\left\{\mathfrak{b}^{0}\right\}$ cells in general position. In other words, these points can be thought of as the vertices of an $(n-3)$-simplex with an additional point at the center. Between every two $\mathfrak{b}^{0}$ points of $\mathcal{B}^{n}$, there exists a $\mathfrak{b}^{1}$ cell, resulting in $\left(\begin{array}{c}n-1 \\ 2\end{array}\right)$ such irreducible lines. In general, $k$ irreducible points of $\mathcal{B}^{n}$ span a $k-1$ dimensional irreducible cell; restating this, we get

\footnotetext{
${ }^{7}$ These copies of $K_{n-1}$ are in bijection with the vertices of the permutohedron $P_{n-1}$ ].
} 
Proposition 5.1.1. The number of the codim $k$ irreducible components of $\mathcal{B}^{n}$ equals

$$
\left(\begin{array}{l}
n-1 \\
k+1
\end{array}\right)
$$

The construction above shows that around a point $\mathfrak{b}^{0} \subset \mathbb{P}\left(V^{n}\right)$, the structure of $\mathcal{B}^{n}$ resembles the barycentric subdivision of an $n-3$ simplex. We look at some concrete examples to demonstrate this.

Example 5.1.2. In the case of $\overline{\mathcal{M}}_{0}^{5}(\mathbb{R})$, Figure 7 shows the $\mathfrak{b}^{0}$ cells in general position; there are four blown up points, three belonging to vertices of a 2-simplex, and one in the center of this simplex. Between every two of these points, there exists a $\mathfrak{b}^{1}$; notice Figure 7 showing 6 such lines. Since these lines are of codim one, they do not need to be blown up.

Looking at the structure of a blown up point $\mathfrak{b}^{0}$ in $\mathcal{B}^{5}$, notice that $\mathfrak{s b}^{0}$ is a hexagon and $\mathfrak{p b}^{0}$ is a triangle. It is no coincidence that these correspond exactly to $\widetilde{\mathcal{M}}_{0}^{4}(\mathbb{R})$ and $\overline{\mathcal{M}}_{0}^{4}(\mathbb{R})$ (see Figure 18).

Example 5.1.3. For the three dimensional $\overline{\mathcal{M}}_{0}^{6}(\mathbb{R})$, the $\mathfrak{b}^{0}$ cells and the $\mathfrak{b}^{1}$ cells need to be blown up, in that order. Choose a codim 3 cell $\mathfrak{b}^{0}$; recall that a neighborhood around $\mathfrak{b}^{0}$ will resemble the barycentric subdivision of a 3 -simplex. Figure 19 shows four tetrahedra, each being made up of six tetrahedra (some shaded), pulled apart in space such that when glued together the result will constitute the aforementioned subdivision. The barycenter is the point $\mathfrak{b}^{0}$.

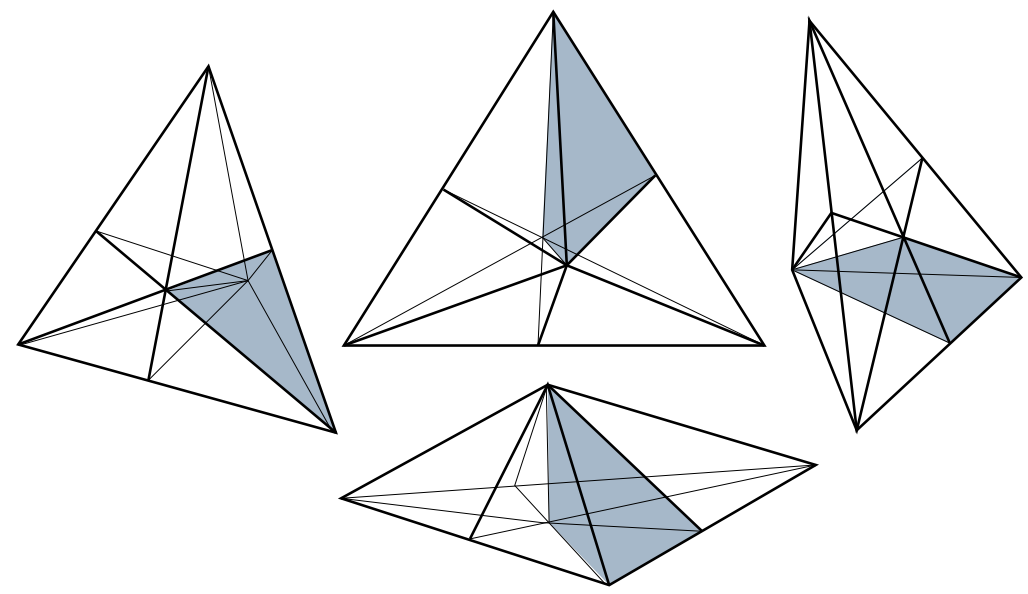

Figure 19. Barycentric subdivision of a 3 -simplex

The left-most piece of Figure 20 shows one of the tetrahedra from Figure 19. The map $f_{1}$ takes the point $\mathfrak{b}^{0}$ to $\mathfrak{s b}^{0}$ whereas the map $f_{2}$ takes each $\mathfrak{b}^{1}$ to $\mathfrak{s b}^{1}$. When looking down at the resulting 'blown up' tetrahedron piece, there are 6 pentagons (shaded) with a hexagon hollowed out in the center. Taking $\mathfrak{s b}^{1}$ to $\mathfrak{p b}^{1}$ turns these hexagons into triangles. Putting the four 'blown up' tetrahedra pieces together, the faces of $\mathfrak{s b}^{0}$ make up a two dimensional sphere tiled by 24 pentagons, with 8 hexagons (with antipodal maps) cut out. This turns out to be $\widetilde{\mathcal{M}}_{0}^{5}(\mathbb{R})$; projectifying $\mathfrak{s b}^{0}$ to $\mathfrak{p b}^{0}$ yields $\overline{\mathcal{M}}_{0}^{5}(\mathbb{R})$ (Figure 21). 

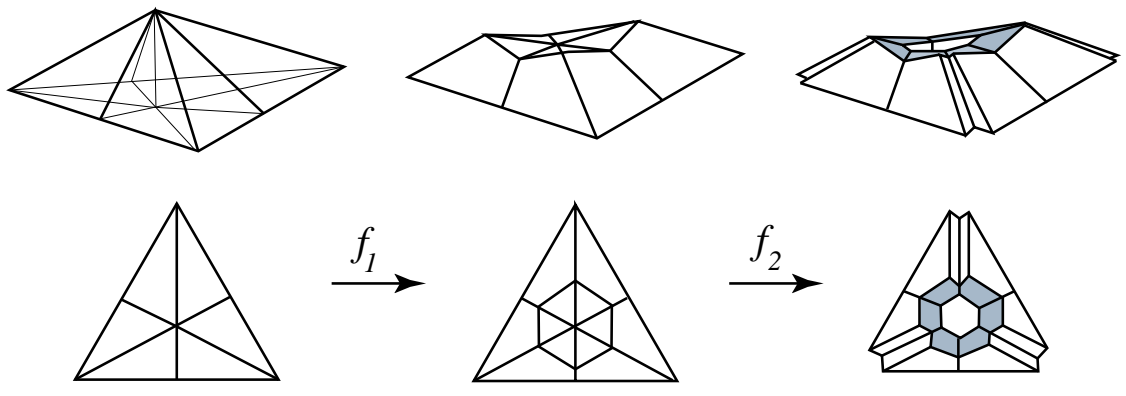

FigURE 20.
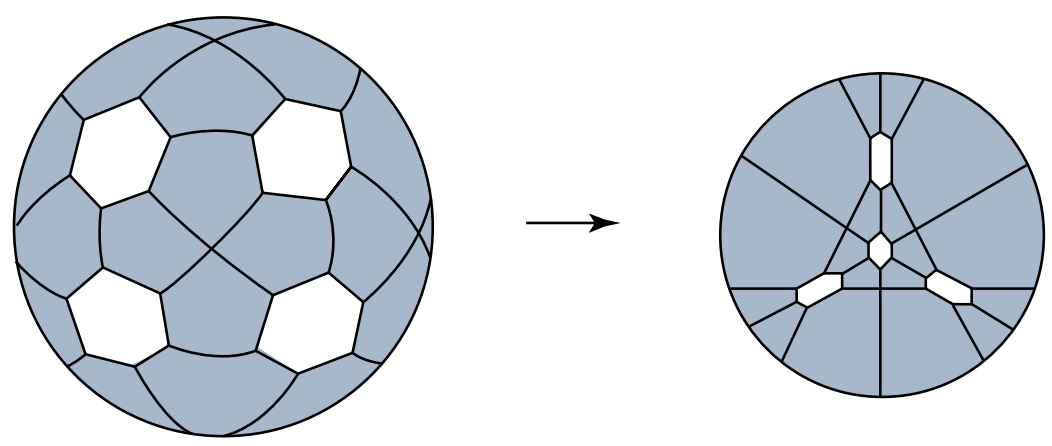

Figure 21. $\tilde{\mathcal{M}}_{0}^{5}(\mathbb{R}) \rightarrow \overline{\mathcal{M}}_{0}^{5}(\mathbb{R})$

This pattern seems to indicate that for $\overline{\mathcal{M}}_{0}^{n}(\mathbb{R})$, blowing up along the $\mathfrak{b}^{0}$ cells will yield $\overline{\mathcal{M}}_{0}^{n-1}(\mathbb{R})$. But what happens in general, when a codim $k$ cell $\mathfrak{b}^{n-3-k}$ is blown up? A glimpse of the answer was seen above with regard to the hexagons and triangles showing up in $\overline{\mathcal{M}}_{0}^{6}(\mathbb{R})$.

5.2. To better understand $\overline{\mathcal{M}}_{0}^{n}(\mathbb{R})$, we look at the faces of the associahedra that surround each blown up component of $\mathcal{B}^{n}$; this is done through the eyes of mosaics. From the remark in $\$ 2.3$ it follows that each blow-up along a codim $k$ cell $\mathfrak{b}^{n-3-k}$ corresponds to a place where $k+1$ marked points on $\mathbb{R} \mathbb{P}^{1}$ collide. The discussion in $\$ 2.1$ relates this to having a diagonal $d$ partition an $n$-gon such that $k+1$ labeled sides $\left\{p_{1}, \ldots, p_{k+1}\right\}$ lie on one side and $\left\{p_{k+2}, \ldots, p_{n}\right\}$ lie on the other. Using the mosaic operad structure, $d$ decomposes the $n$-gon into $G_{1} \circ G_{2}$, where $G_{1} \in \mathcal{G}^{L}(k+2)$ and $G_{2} \in \mathcal{G}^{L}(n-k)$, with the new sides $d_{i}$ of $G_{i}$ coming from $d$. Note that $G_{1} \circ G_{2}$ corresponds to the product of associahedra $K_{k+1} \times K_{n-k-1}$.

Fix a particular labeling of $G_{2}$ using the elements $\left\{p_{k+2}, \ldots, p_{n}\right\}$. There are $(k+1)$ ! different ways in which $\left\{p_{1}, \ldots, p_{k+1}\right\}$ can be arranged to label $G_{1}$. However, since twisting is allowed along $d_{1}$, we get $\frac{1}{2}(k+1)$ ! different labelings of $G_{1}$, each corresponding to a $K_{k+1}$. But observe that this is exactly how one gets $\overline{\mathcal{M}}_{0}^{k+2}(\mathbb{R})$, where the associahedra glue as defined in $\$ 4.1$. Therefore, for a fixed labeling of $G_{2}$, we get $\overline{\mathcal{M}}_{0}^{k+2}(\mathbb{R}) \times K_{n-k-1}$; looking at all possible labelings gives 
Theorem 5.2.1. Blowing up along a codim $k$ cell $\mathfrak{b}^{n-3-k}$ of $\overline{\mathcal{M}}_{0}^{n}(\mathbb{R})$ results in

$$
\overline{\mathcal{M}}_{0}^{k+2}(\mathbb{R}) \times \overline{\mathcal{M}}_{0}^{n-k}(\mathbb{R}) .
$$

Notice how this was obtained for a particular partition of $\left\{p_{1}, \ldots, p_{n}\right\}$. Therefore, the number of places that (5.2) occurs becomes

$$
\left(\begin{array}{c}
n \\
k+1
\end{array}\right)
$$

corresponding to partitioning $\left\{p_{1}, \ldots, p_{n}\right\}$ into two sets of order $k+1$ and $n-k-1$. However, (5.3) alone does not tell the whole story since it fails to match up with the result of (5.1). This is due to a sense of 'duality' that is present: The construction above is unable to distinguish between the spaces $\overline{\mathcal{M}}_{0}^{k+2}(\mathbb{R}) \times \overline{\mathcal{M}}_{0}^{n-k}(\mathbb{R})$ and $\overline{\mathcal{M}}_{0}^{n-k}(\mathbb{R}) \times \overline{\mathcal{M}}_{0}^{k+2}(\mathbb{R})$. Since

$$
\left(\begin{array}{c}
n \\
k+1
\end{array}\right)=\left(\begin{array}{l}
n-1 \\
k+1
\end{array}\right)+\left(\begin{array}{c}
n-1 \\
n-k-1
\end{array}\right)
$$

we see (5.3) actually gives the sum of the number of codim $k$ and codim $n-k-2$ components. By fixing one of the $n$ points to be $\infty$, we see through this duality: Replacing the codim $k$ cell $\mathfrak{b}^{n-3-k}$ of $\overline{\mathcal{M}}_{0}^{n}(\mathbb{R})$ with $\mathfrak{s b}^{n-3-k}$ now results in

$$
\tilde{\mathcal{M}}_{0}^{k+2}(\mathbb{R}) \times \overline{\mathcal{M}}_{0}^{n-k}(\mathbb{R}) .
$$

Example 5.2.2. The blown up $\mathfrak{b}^{0}$ cells yields $\widetilde{\mathcal{M}}_{0}^{n-1}(\mathbb{R})$ for $\mathfrak{s} \mathfrak{b}^{0}$ and $\overline{\mathcal{M}}_{0}^{n-1}(\mathbb{R})$ for $\mathfrak{p b}^{0}$, matching the earlier observations of $\$$ 5.1. Furthermore, (5.1) shows there to be $n-1$ such structures.

Example 5.2.3. Although blowing up along codim one components does not affect the resulting manifold, we observe their presence in $\overline{\mathcal{M}}_{0}^{5}(\mathbb{R})$. From (5.1), we get six such $\mathfrak{b}^{n-4}$ cells; these turn out to be $\widetilde{\mathcal{M}}_{0}^{3}(\mathbb{R}) \times \overline{\mathcal{M}}_{0}^{4}(\mathbb{R})$ for $\mathfrak{s b}^{n-4}$ and $\overline{\mathcal{M}}_{0}^{4}(\mathbb{R})$ for $\mathfrak{p b}^{n-4}$. The $\overline{\mathcal{M}}_{0}^{4}(\mathbb{R})$ 's are seen in Figure 7 as the six lines cutting through $\mathbb{R} \mathbb{P}^{2}$. Note that every line is broken into six parts, each part being a $K_{3}$.

Example 5.2.4. The space $\overline{\mathcal{M}}_{0}^{6}(\mathbb{R})$, illustrated in Figure 9, moves a dimension higher. . There are ten $\mathfrak{b}^{1}$ cells, each having $\widetilde{\mathcal{M}}_{0}^{4}(\mathbb{R}) \times \overline{\mathcal{M}}_{0}^{4}(\mathbb{R})$ for $\mathfrak{s b}^{1}$. These are the hexagonal prisms that cut through the three torus as described in Example 2.3.3.

5.3. The question arises as to why $\overline{\mathcal{M}}_{0}^{n-k}(\mathbb{R})$ appears in $\overline{\mathcal{M}}_{0}^{n}(\mathbb{R})$. The answer lies in the braid arrangement of hyperplanes. Taking $\overline{\mathcal{M}}_{0}^{6}(\mathbb{R})$ as an example, blowing up along each $\mathfrak{b}^{0}$ in $\mathcal{B}^{6}$ uses the following procedure: A small spherical neighborhood is drawn around $\mathfrak{b}^{0}$ and the inside of the sphere is removed, resulting in $\mathfrak{s b}^{0}$. Observe that the sphere (which we denote as $\mathcal{S}$ ) is engraved with great arcs coming from $\mathcal{B}^{6}$. Projectifying, $\mathfrak{s b}^{0}$ becomes $\mathfrak{p b}^{0}$, and $\mathcal{S}$ becomes the projective sphere $\mathbb{P} \mathcal{S}$. Amazingly, the engraved arcs on $\mathbb{P S}$ are $\mathcal{B}^{5}$, and $\mathbb{P S}$ can be thought of as $\mathbb{P}\left(V^{5}\right)$. Furthermore, blowing up along the cells $\mathfrak{b}^{1}$ of $\mathcal{B}^{6}$ corresponds to blowing up along the cells $\mathfrak{b}^{0}$ of $\mathcal{B}^{5}$ in $\mathbb{P S}$ ! As before, this new etching on $\mathbb{P S}$ translates into an even lower dimensional braid arrangement, $\mathcal{B}^{4}$.

${ }^{8}$ Although this figure is not constructed from the braid arrangement, it is homeomorphic to the structure described by the braid arrangement. 
It is not hard to see how this generalizes in the natural way: For $\overline{\mathcal{M}}_{0}^{n}(\mathbb{R})$, the iterated blow-ups along the cells $\left\{\mathfrak{b}^{0}\right\}$ up to $\left\{\mathfrak{b}^{n-5}\right\}$ in turn create braid arrangements within braid arrangements. Therefore, $\overline{\mathcal{M}}_{0}^{n-k}(\mathbb{R})$ is seen in $\overline{\mathcal{M}}_{0}^{n}(\mathbb{R})$.

\section{The Fundamental Group}

6.1. Coming full circle, we look at the connections between the results from the little cubes and the mosaic operads.

Definition 6.1.1. [4, §3] Let $J_{n-1}$ to be the right-angled Coxeter group of $K_{n-1}$. That is, $J_{n-1}$ is generated by reflections $\left\{s_{i}\right\}$ which correspond bijectively to the codim one faces $\left\{\lambda_{i}\right\}$ of $K_{n-1}$, where $s_{i} s_{j}=s_{j} s_{i}$ if and only if $\lambda_{i} \cap \lambda_{j} \neq \emptyset$.

Let $G \in \mathcal{G}^{L}(n, 0)$ be an $n$-gon having sides with the cyclic labeling $\{1,2, \ldots, n\}$. Define $G_{\lambda} \in \mathcal{G}^{L}(n, 1)$ to be the set $\left\{g_{i}\right\}$ of polygons $G$ with one diagonal; Lemma 4.2.1 shows the order of $G_{\lambda}$ to be $\frac{1}{2}\left(n^{2}-3 n\right)$. From $\$ 3.2$, we have the following bijections:

$$
\left\{s_{i}\right\} \longleftrightarrow\left\{\lambda_{i}\right\} \longleftrightarrow\left\{g_{i}\right\}
$$

For $g_{1}, g_{2} \in G_{\lambda}$, create a new polygon (with two diagonals) by superimposing the images of $g_{1}$ and $g_{2}$ on each other such that the labelings match (Figure 22). Two elements of $G_{\lambda}$ commute (as images in $J_{n-1}$ ) if and only if the diagonals of their superimposed polygons do not intersect. Furthermore, if the two elements do commute, the codim two cell of intersection in $K_{n-1}$ corresponds to the superimposed polygons. This follows immediately from the properties of the associahedron.
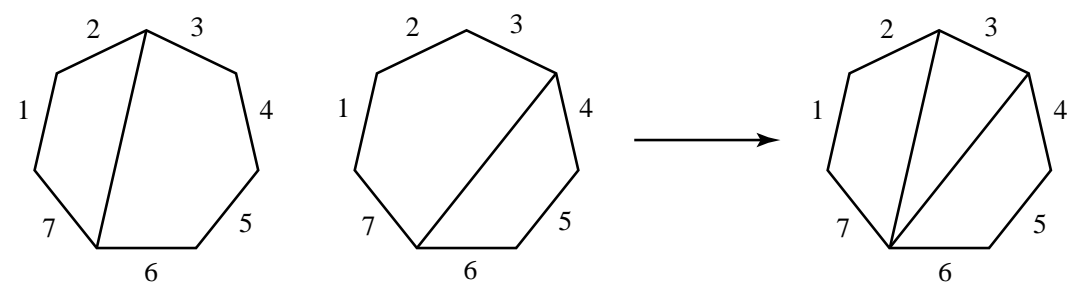

FiguRE 22. Superimpose

The Coxeter group $J_{n-1}$ is used to better understand the homotopy groups of $\overline{\mathcal{M}}_{0}^{n}(\mathbb{R})$. However, Davis, Januszkiewicz, and Scott have shown in the following theorem that the homotopy properties of $\overline{\mathcal{M}}_{0}^{n}(\mathbb{R})$ are completely encapsulated in its fundamental group.

Theorem 6.1.2. [5, $\S 5.1] \overline{\mathcal{M}}_{0}^{n}(\mathbb{R})$ is aspherical.

6.2. We now introduce a conjecture of Januszkiewicz, which together with the machinery above is used to analyze $\pi_{1}\left(\overline{\mathcal{M}}_{0}^{n}(\mathbb{R})\right)$.

Conjecture 6.2.1. [7] When $n$ exceeds 3 ,

$$
\pi_{1}\left(\overline{\mathcal{M}}_{0}^{n}(\mathbb{R})\right) \succ D_{n} \ltimes J_{n-1} \rightarrow \mathbb{S}_{n} .
$$

Remark. By Proposition 3.2.4, the dihedral group $D_{n}$ acts on $K_{n-1}$. From (6.1), it follows that $D_{n}$ acts on $J_{n-1}$, preserving the relations. 

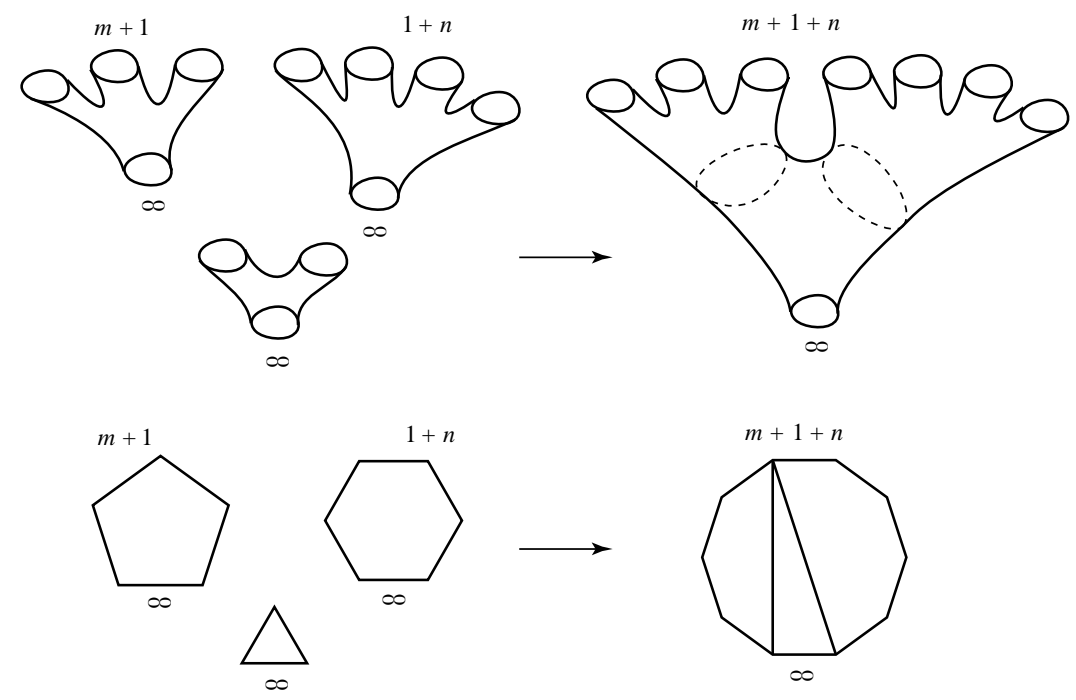

Figure 23. Pair-of-pants

Consider the subset $G_{*} \subset G_{\lambda}$ whose diagonals partition the sides of the $n$ gon into a set of order two (say with labels $a$ and $b$ ) and of order $n-2$. Let $\phi: G_{\lambda} \rightarrow \mathbb{S}_{n}$ be defined as follows: Elements of $G_{*}$ are mapped to the transposition $(a, b)$; the rest of $G_{\lambda}$ is mapped to the identity. To check that this map is welldefined, we must verify that the commutativity property of $G_{\lambda}$ (coming from $J_{n-1}$ ) must hold in $\phi\left(G_{\lambda}\right)$. Since $\phi$ maps $G_{\lambda}-G_{*}$ to the identity, we need only restrict to elements of $G_{*}$. As noted above, elements of $G_{*}$ commute only when their superimposed polygon has non-intersecting diagonals. But this is only possible when the corresponding partitioned labels $\left\{a_{1}, b_{1}\right\}$ and $\left\{a_{2}, b_{2}\right\}$ are all distinct. It follows that the transposition elements $\left(a_{1}, b_{1}\right)$ and $\left(a_{2}, b_{2}\right)$ commute in $\mathbb{S}_{n}$. The bijection of (6.1) extends to define a homomorphism $\phi: J_{n-1} \rightarrow \mathbb{S}_{n}$.

Conjecture 6.2.2. The map taking $J_{n-1}$ to $\mathbb{S}_{n}$ in (6.2) is $\phi$.

Let $G$ be the $n$-gon with cyclic labeling $\{1,2, \ldots, n\}$ defined above. As $D_{n}$ acts on $G$, it permutes the $n$ labels; therefore, there is a natural inclusion $i: D_{n} \hookrightarrow \mathbb{S}_{n}$. For elements $d \in D_{n}$ and $c \in J_{n-1}$, let $\psi$ be the map $D_{n} \ltimes J_{n-1} \rightarrow \mathbb{S}_{n}$ of (6.2) defined as follows: $\psi(d, c)=i(d) \cdot \phi(c)$. This map is surjective since the transpositions $\{(1,2), \ldots,(n-1, n),(n, 1)\}$ generate $\mathbb{S}_{n}$. It immediately follows that

$$
\operatorname{ker} \psi=\left\{\left(\phi\left(c^{-1}\right), c\right) \mid c \in \phi^{-1}\left(D_{n}\right)\right\}=\phi^{-1}\left(D_{n}\right) .
$$

Therefore, $\pi_{1}\left(\overline{\mathcal{M}}_{0}^{n}(\mathbb{R})\right)$ is isomorphic to $\phi^{-1}\left(D_{n}\right)$.

6.3. There exists a natural pullback of $\mathbb{S}_{n+1}$ to $\mathbb{S}_{n}$ by fixing of one the $n+1$ points to be $\infty$, thereby breaking symmetry. Using (6.2), the pullback of $D_{n+1} \ltimes J_{n}$ fits into the following commutative diagram:

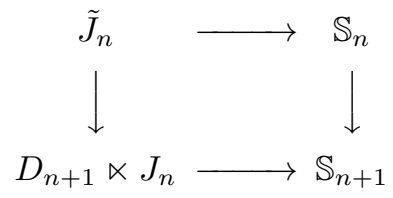


The pair-of-pants product (Figure 23) takes $m+1$ and $1+n$ marked points on $\mathbb{R} \mathbb{P}^{1}$ to $m+1+n$ marked points. The operad structure on the spaces $\overline{\mathcal{M}}_{0}^{n+1}(\mathbb{R})$, its simplest case corresponding to the pair-of-pants product, defines composition maps

$$
\tilde{J}_{m} \times \tilde{J}_{n} \rightarrow \tilde{J}_{m+n}
$$

analogous to the juxtaposition map of braids. We can thus construct a monoidal category which has finite ordered sets as its objects and the group $\tilde{J}_{n}$ as the automorphisms of a set of cardinality $n$, all other morphism sets being empty. Note the following similarity between the braid group $\mathbf{B}_{n}$ obtained from the little cubes operad and the 'quasibraids' $\tilde{J}_{n}$ obtained from the mosaics operad:

$$
\begin{aligned}
& \pi_{1}\left(\mathbb{C}^{n}-\Delta\right) \quad \longmapsto \mathbf{B}_{n} \rightarrow \mathbb{S}_{n} \\
& \pi_{1}\left(\overline{\left(\left(\mathbb{R P}^{1}\right)^{n+1}-\Delta\right) / \mathbb{P G l}_{2}(\mathbb{R})}\right) \quad \longmapsto \quad \tilde{J}_{n} \quad \rightarrow \quad \mathbb{S}_{n}
\end{aligned}
$$

There are deeper analogies between these structures which have yet to be studied.

\section{REFERENCES}

1. H. R. Brahana, A. M. Coble, Maps of twelve countries with five sides with a group of order 120 containing an Ikosahedral subgroup, Amer. J. Math. 48 (1926) 1-20.

2. J. M. Boardman, R. M. Vogt, Homotopy invariant algebraic structures on topological spaces, Lecture Notes in Math. 347 (1973).

3. A. Cayley, On the partitions of a polygon, Proc. Lond. Math. Soc. 22 (1890-91) 237-262

4. R. Charney, M. Davis, Finite $K(\pi, 1)$ 's for Artin Groups, Prospects in Topology (ed. F. Quinn), Annals of Math. Studies 138 (1995) 110-124.

5. M. Davis, T. Januszkiewicz, R. Scott, Nonpositive curvature of blowups, Ohio State preprint 97-3.

6. E. Getzler, M. M. Kapranov, Cyclic operads and cyclic homology, Geometry, Topology, and Physics for Raoul Bott, ed. S. T. Yau, International Press (1994).

7. T. Januszkiewicz, Private communication with Jack Morava.

8. M. M. Kapranov, Chow quotients of Grassmannians. I, Adv. in Sov. Math. 16 (1993) 29-110.

9. M. M. Kapranov, The permutoassociahedron, Mac Lane's coherence theorem, and asymptotic zones for the KZ equation, J. Pure Appl. Alg. 85 (1993) 119-142.

10. S. Keel, Intersection theory of moduli spaces of stable n-pointed curves, Trans. Amer. Math. Soc. 330 (1992) 545-574.

11. M. Kontsevich, Y. Manin, Gromov-Witten classes, quantum cohomology, and enumerative geometry, Comm. Math. Phys. 164 (1994) 525-562.

12. C. Lee, The associahedron and triangulations of the n-gon, European J. Combin. 10 (1989) 551-560.

13. J. P. May, The geometry of iterated loop spaces, Lecture Notes in Math. 271 (1972)

14. J. P. May, Definitions: operads, algebras and modules, Contemp. Math. 202 (1997) 1-7.

15. J. G. Ratcliffe, Foundations of hyperbolic manifolds, Grad. Text. in Math. 149, SpringerVerlag (1994).

16. J. D. Stasheff (Appendix B coauthored with S. Shnider), From operads to 'physically' inspired theories, Contemp. Math. 202 (1997) 53-81.

17. J. D. Stasheff, Homotopy associativity of H-spaces I, Trans. Amer. Math. Soc. 108 (1963) 275-292.

18. M. Yoshida, Hypergeometric functions, my love, Vieweg (1997).

19. G. M. Ziegler, Lectures on polytopes, Grad. Text. in Math. 152, Springer-Verlag (1995).

Department of Mathematics, Johns Hopkins University, Baltimore, Maryland 21218

E-mail address: devadoss@math.jhu.edu 